\title{
The mechanical characterization of stacked, multilayer graphene cantilevers and plates
}

\author{
Emil Sandoz-Rosado, Eric D. Wetzel* \\ Materials and Manufacturing Sciences Division \\ Army Research Laboratory \\ Aberdeen Proving Ground, MD, USA \\ *eric.d.wetzel2.civ@mail.mil
}

\author{
Joshua T. Smith, Satoshi Oida, Jingwei Bai \\ Watson Research Center \\ IBM \\ Yorktown Heights, NY USA
}

\begin{abstract}
The mechanical properties of stacked graphene sheets with varying number of layers are examined. The stacked sheets are assembled by manually combining single layer CVDgrown graphene monolayers, resulting in a turbostratic multilayer graphene with irregular layer spacing greater than crystalline graphite. Due to the presence of multiple layers, the material is analyzed as a plate rather than a membrane. Bending stiffness is determined via the deflection of micron-scale cantilevers, prepared using focused ion beam milling, while inplane tensile stiffness is characterized through center-loading of edge-supported circular specimens. Computational modeling and established analytical solutions are used to extract material and structural property information, and benchmark measured properties relative to complementary results from indentation tests. Stacked, few-layer CVD-grown graphene retains an inplane elastic modulus of $350 \mathrm{~N} / \mathrm{m} /$ layer (corresponding to 1.04 TPa for an inter-layer spacing of $0.335 \mathrm{~nm}$ ), suggesting good loadsharing between stacked layers. Width-normalized bending stiffness was unmeasurable for cantilevers of 1 and 3 layers, while cantilevers of 5 and 10 layers had values of $11,100 \mathrm{nN} \cdot \mathrm{nm}$ and $1.3 \cdot 10^{6} \mathrm{nN} \cdot \mathrm{nm}$ respectively.
\end{abstract}

Keywords-graphene; mechanical; plate; cantilever; elastic; bending (key words)

\section{INTRODUCTION}

The in-plane tensile elastic modulus of graphene, reported at $1 \mathrm{TPa}$ for both CVD-grown granular graphene[1] and exfoliated graphene[2], makes graphene one of the stiffest materials known to exist. The intrinsic strength of graphene is estimated to be much greater than most engineering polymers, metals, and composites, making it a potentially ideal structural material. However, the scaling of graphene's mechanical properties with increased number of layers has yet to be fully explored.

A common approach for quantifying monolayer and few-layer graphene is to center-load an edge-supported circular graphene membrane. The primitive load-deflection data is then analyzed using a semi-empirical membrane model in order to extract an in-plane elastic modulus value[2]. This approach assumes that the few-layer graphene stack behaves as a pure membrane, without bending stiffness. However, as the graphene stack reaches 3 or 4 layers, the bending stiffness cannot be neglected and a plate model is needed[3, 4]. A plate model assuming infinitely small beam bending rotations has been implemented for experimental characterization of a graphene flake 69 layers thick, although this approach is not applicable to few-layer graphene for which significant rotations during loading are expected[5]. An appropriate analysis approach for a thin plate with finite bending rotations, such as for a few-layer graphene stack, is a von Karman plate model[6]. The von Karman plate model has been applied to atomistic simulations but has yet to be employed for the purposes of interpreting the mechanical response of few-layer graphene.

Few studies have been performed to directly characterize the bending stiffness of a graphene stack. In one study the authors found a single cantilevered protrusion of graphene and performed AFM loading to quantify its bending stiffness, but did not attempt to systematically explore this property over many samples[7]. The effect of factors such as number of layers and crystallographic stacking registration on bending stiffness is not well understood. In the present study, we demonstrate the mechanical loading of graphene cantilevers as a means of generating bending stiffness without in-plane tensile loading. These measured bending stiffness values are then used to improve our characterization of centerloaded circular plate experiments, in which both in-plane stresses and bending stresses are significant.

\section{EXPERIMENT}

\section{A. Graphene Stacking and Transfer}

Graphene for the various experiments was prepared using CVD growth on copper foil (Alpha Aesar 99.999\% elemental purity) coupled with a wet transfer technique. Single-layer growth was confirmed with Raman spectroscopy by mapping $2 \mathrm{D}$ to $\mathrm{G}$ peak intensity ratios over randomly selected growth areas. In order to minimize interlayer polymer residue, a direct layer-by-layer (LBL) transfer method was adopted to stack and transfer the graphene layers ${ }^{[8]}$. The steps of this stacking process are highlighted in Fig. 1. First, monolayer graphene was grown on copper foil for each individual layer to be added into the overall laminate stack. To create the base layer, a PMMA coating was spun onto the graphene and the copper was etched away. In order to add further layers, a new sheet of graphene attached to its $\mathrm{Cu}$ growth foil was placed into contact with the exposed graphene 


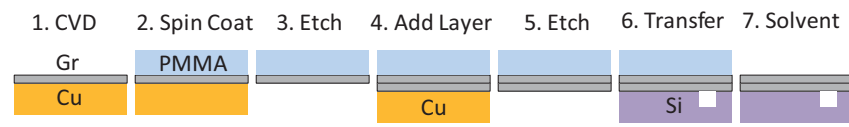

Fig. 1. Graphene LBL stacking process and transfer to silicon wafer with wells for indentation. Steps $4-5$ can be repeated as many times as necessary to achieve the desired number of layers.

of the base layer (on PMMA) using the scooping method. The copper on the new layer of graphene was then etched away. To compare the impact of etchants, one batch of graphene was prepared with ferric chloride etchant and one batch was prepared with ammonium persulfate etchant[1]. This process was repeated until the desired number of layers was achieved. Once the graphene was finished being stacked, it was transferred by floating the PMMA with the graphene stack in de-ionized (DI) water and scooping it onto a silicon wafer with the necessary wells and trenches. The PMMA was then dissolved and cleaned off the graphene by submerging and soaking the stack in acetone, isopropyl alcohol and finally a DI water rinse bath.

\section{B. Graphene Cantilever Fabrication and Testing}

The cantilevers were fabricated by suspending stacked graphene over trenches etched into the silicon wafer and cutting the suspended graphene into cantilevers using FIB milling. By using a narrow beam width, it is possible to cut cantilevers with micron-scale dimensions that could fully support themselves while suspended over the trench (Fig. 2). Graphene cantilevers of 1, 3,5 and 10 layers were prepared and examined.

Cantilevers were deflected using an AFM to extract mechanical properties. A silicon AFM tip with a cantilever stiffness of $20 \mathrm{~N} / \mathrm{m}$ (nominal radius of $7 \mathrm{~nm}$ ) was used for the cantilever experiments because of the low relative stiffness of the graphene cantilever. Using Timoshenko beam theory, the stiffness of a cantilever can be measured as:

$$
D_{c}=\frac{k L^{2}}{18}\left(3 L_{0}-L\right)=E I=\frac{E b h^{3}}{12}=\gamma b
$$

where $k$ is the spring constant measured from the graphene cantilever, $L$ is the position on the cantilever where indentation occurs, and $L_{0}$ is the length of the cantilever. Stiffness can also be re-written in terms of elastic modulus, $E$, and moment of inertia, $I$, which in turn is a function of beam thickness, $h$, and width $b$, or a width-normalized bending stiffness parameter, $\gamma$ (this parameter is useful for comparing cantilever stiffness to plate stiffness measurements).

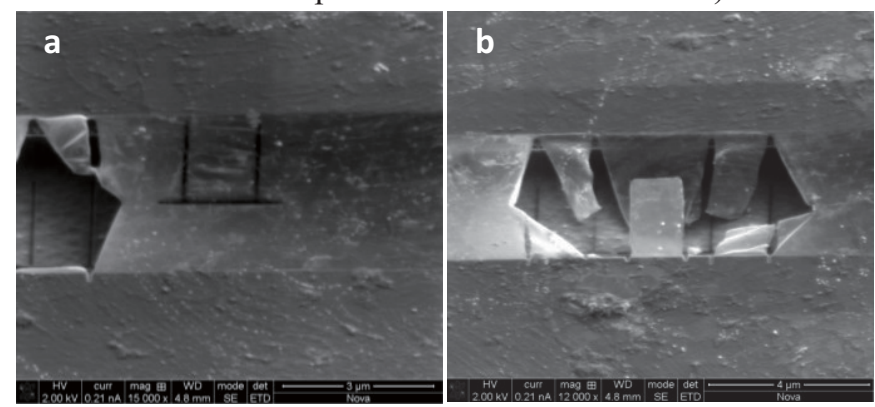

Fig.2. SEM scan of five-layer graphene a) being cut with a FIB and b) finally processed into a cantilever for mechanical testing.

\section{Circular Well Indentation Mechanical Testing}

The prepared graphene stacks were suspended over $1 \mu \mathrm{m}$ and $5 \mu \mathrm{m}$ diameter wells that had been etched into the substrate (300nm thick $\mathrm{SiO}_{2}$ on $\mathrm{Si}$ wafer) to allow for mechanical testing. For monolayer and 3-layer graphene, the graphene membrane is flat and positioned slightly below the wafer surface, due to long-range attractive forces that pull the membrane into contact with the inner walls of the well (see Fig. 3a). Inward or outward buckling occurred for 5 and 10 layer suspended graphene (Fig. 3b). The mechanical properties were identical between inward and outward buckled graphene, with the outward-buckled graphene snapping inward after the first indentation and reproducing the same mechanical response as the other wells on subsequent indents. An atomic force microscope with a diamond tip (nominal radius of $25 \mathrm{~nm}$ ) on a sapphire cantilever (spring constant of $600 \mathrm{~N} / \mathrm{m}$ ) was used to load the center of graphene suspended over circular wells to test the mechanical response.

An axisymmetric von Karman plate model is used to extract mechanical properties from the indentation forcedisplacement curves of the suspended graphene. The coupled axisymmetric plate eq.s are[6]:

$$
\begin{gathered}
\frac{d^{3} w}{d r^{3}}+\frac{1}{r} \frac{d^{2} w}{d r^{2}}-\left(\frac{1}{r^{2}}+\frac{\sigma_{0}+\sigma_{r}}{D_{p}}\right) \frac{d w}{d r}=\frac{F}{2 \pi r D_{p}} \\
\frac{d^{2} \sigma_{r}}{d r^{2}}+\frac{3}{r} \frac{d \sigma_{r}}{d r}=\frac{(1-v)}{2\left(1-v^{2}\right)} \frac{E_{2 D}}{r^{2}}\left(\frac{d w}{d r}\right)^{2}
\end{gathered}
$$

where $w$ is transverse deflection of the center point, $r$ is the radial direction, $\sigma_{r}$ is tensile stress in the radial direction, $F$ is the point load at the center, $v$ is the Poisson ratio, $E_{2 D}$ is the inplane, 2-D elastic modulus, $\sigma_{0}$ is the prestress, and $D_{p}$ is plate bending stiffness.

We used the Lobatto algorithm to solve the von Karman eq.s computationally[6] to curve fit (through Simplex minimization) experimental force-distance plots by matching $w$ and $F$ to experiment, prescribing $v$ (value of 0.16 for graphene/graphite) and tuning $E_{2 D}$ and $\sigma_{0}$ as fitting parameters. Plate bending stiffness, $D_{p}$, is known from cantilever experiments on a corresponding graphene sample, and can be related to the width-normalized cantilever bending stiffness $\gamma$ in Eq. 1 according to:

$$
D_{p}=\frac{E h^{3}}{12\left(1-v^{2}\right)}=\frac{\gamma}{\left(1-v^{2}\right)}
$$
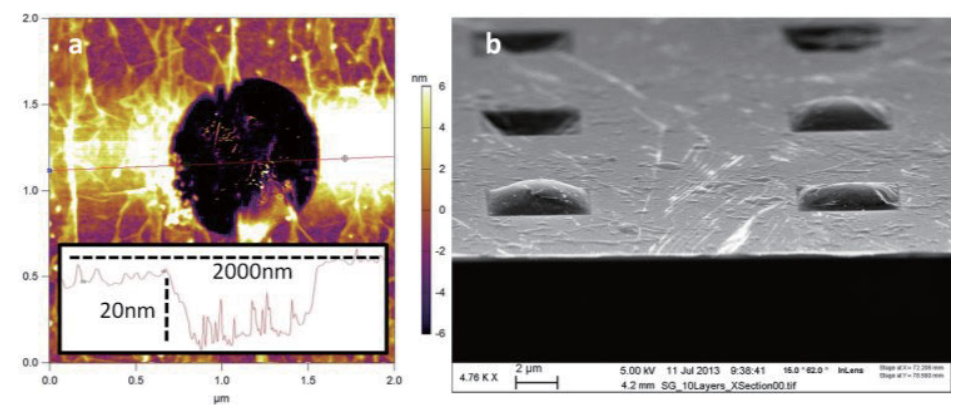

Fig. 3. a) AFM scan of three-layer graphene suspended over a $1 \mu \mathrm{m}$ diameter hole with a profile inlay and b) SEM image of ten-layer graphene suspended over 5 um square wells depicting buckling behavior. 

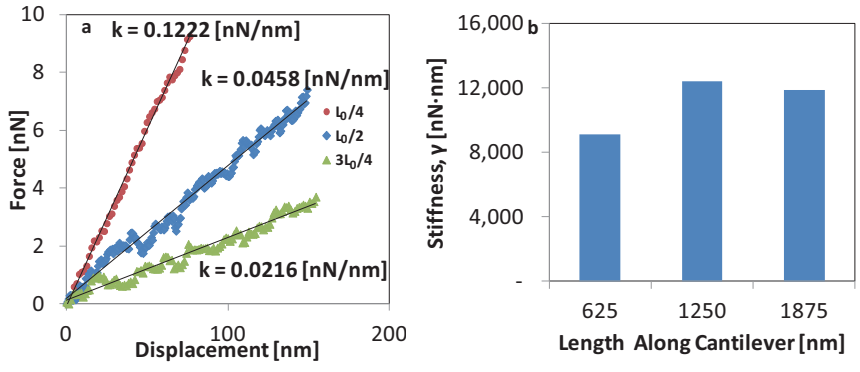

Fig. 4. a) Force-displacement curves for a 5-layer $2.5 \mu \mathrm{m} \times 2.0 \mu \mathrm{m}$ graphene cantilever and b) width-normalized stiffness, $\gamma$, as a function of cantilever length.

\section{RESUltS AND DISCUSSION}

To ensure the Timoshenko cantilever model (Eq. 1) was adequate, a 5-layer ferric-chloride-processed graphene cantilever with dimensions $2.5 \mu \mathrm{m} \times 2.0 \mu \mathrm{m}$ was loaded at several points along the cantilever length (Fig. 4). At 1/4, 1/2, and $3 / 4$ of the overall length of the cantilever $\left(L_{0}=2.5 \mu \mathrm{m}\right)$, and always along the center axis of the cantilever. The widthnormalized bending stiffness only varied by $16 \%$ from the average, indicating that the graphene protrusions we processed are sufficiently rigid to be modeled as a cantilever.

The 5-layer ferric-chloride-processed cantilever in Fig. 4 had an average normalized stiffness of $11,100 \mathrm{nN} \cdot \mathrm{nm}$. Using this bending stiffness value, the resulting plate model fit (Eqns. 2-3) to experimental center-loading data for a 5-layer sample suspended over a $1 \mu \mathrm{m}$ diameter well yields a per-layer stiffness of $E_{2 D}=250 \mathrm{~N} / \mathrm{m} /$ layer. For comparison, we performed center loading experiments on a 3-layer sample and a monolayer sample, in both cases prepared with ferricchloride etchant and mounted over $1 \mu \mathrm{m}$ diameter wells, and fit the data using a traditional two-parameter membrane model ${ }^{2}$. The resulting in-plane stiffness values were $E_{2 D}=260$ $\mathrm{N} / \mathrm{m} /$ layer and $275 \mathrm{~N} / \mathrm{m} /$ layer, respectively. The general consistency of the per-layer in-plane stiffness values supports the validity of our cantilever measurements, while the trend of slightly decreasing in-plane stiffness with increasing layers could be reflective of damage during stacking or increased out-of-plane folding and buckling.

The measured normalized stiffness of ferric-chlorideprocessed graphene was $11,100 \mathrm{nN} \cdot \mathrm{nm}$ for 5-layer and $1.3 \times 10^{6} \mathrm{nN} \cdot \mathrm{nm}$ for 10 -layer cantilevers. Although the stiffness value should scale as the number of layers cubed, the 10-layer is much stiffer than expected. This difference may be due to the layer spacing being larger than anticipated, corrugation effects, or imperfect processing. Cantilevers with 1 or 3 layers did not survive AFM indentation, so stiffness values are not reported. We intend to extract mechanical properties for 1 or 3 layer graphene cantilevers using a less-stiff AFM cantilever.

To examine the effect of etchant, 3-layer graphene processed with ammonium persulfate etchant was indented to the point of failure over seven independent $5 \mu \mathrm{m}$ diameter wells and the results were curve fit with the von Karman plate eq.s (Fig. 5a). For our 3-layer graphene indentation experiment, cantilever data is not presently available so both $E_{2 D}$ and $D_{p}$ were fit as independent variables, yielding $E_{2 D}=$ $350 \mathrm{~N} / \mathrm{m} /$ layer and a normalized stiffness of $\gamma=$
$33,500 \mathrm{nN} \cdot \mathrm{nm}$. The in-plane stiffness value is in good agreement with previously-reported experimental measurements of $340 \mathrm{~N} / \mathrm{m} /$ layer[2]. For comparison, the 3layer graphene processed with ferric chloride had an $E_{2 D}$ value of $260 \mathrm{~N} / \mathrm{m} /$ layer, indicating that graphene's mechanical properties are negatively impacted by the ferric chloride. While previous studies have shown that breaking strength can be impacted by etchant[1], the discrepancy in stiffness requires further study.

\section{CONCLUSIONS}

The results of this study suggest that the cantilever and circular well experiments are excellent complementary experimental methods for mechanical characterization of stacked graphene, reducing the number of unknown parameters and producing results consistent with prior literature values. The few-layer graphene prepared with ammonium persulfate etchant had in-plane elastic modulus of $350 \mathrm{~N} / \mathrm{m} /$ layer, which is in good agreement with theory and experimentation for pristine graphene. The general agreement fo in-plane modulus between stacked CVD-grown graphene and pristine graphene suggests both good quality and good load-sharing between stacked layers. Bending stiffness (normalized by cantilever width) was not successfully measured for cantilevers of 1 and 3 layers, while cantilevers of 5 and 10 layers had values of $11,100 \mathrm{nN} \cdot \mathrm{nm}$ and $1.3 \times 10^{6} \mathrm{nN} \cdot \mathrm{nm}$ respectively. The bending stiffness increased at a rate larger than the expected $N^{3}$ (where $N$ is number of layers), suggesting that inter-layer spacing for stacked graphene is larger than the crystalline stacking distance of $0.335 \mathrm{~nm}$. The slight decrease in $E_{2 D}$ with increasing layer count, as measured for monolayer, 3-layer, and 5-layer ferricchloride-etched samples, could be due to systematic damage from the aggressive etchant, or could be a insignificant trend that will disappear with increased sample count. Tests are underway using higher quality samples, released with a lessdamaging ammonium persulfate etchant, and with larger sample populations to generate statistically significant data sets.
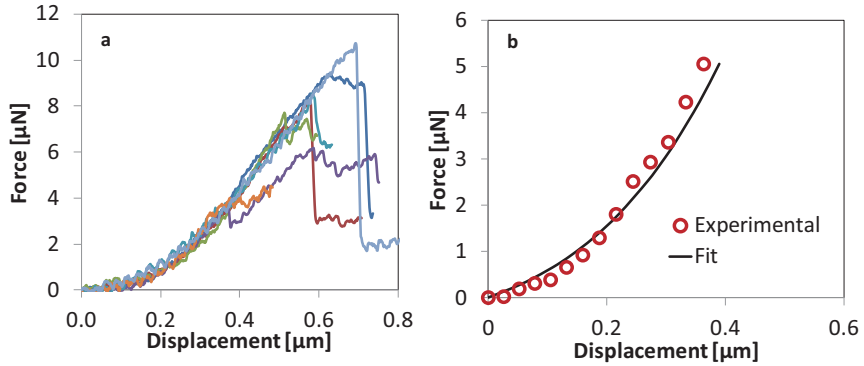

Fig. 5. a) Indentation to failure for three-layer graphene over seven independent $5 \mu \mathrm{m}$ diameter wells and b) curve fit of the three-layer graphene. 


\section{REFERENCES}

[1] G.-H. Lee, R. C. Cooper, S. J. An, S. Lee, A. van der Zande, N. Petrone, A. G. Hammerberg, C. Lee, B. Crawford, W. Oliver, J. W. Kysar, and J. Hone, "High-Strength Chemical-VaporDeposited Graphene and Grain Boundaries," Science, vol. 340, pp. 1073-1076, May 31, 2013.

[2] C. Lee, X. Wei, J. W. Kysar, and J. Hone, "Measurement of the elastic properties and intrinsic strength of monolayer graphene," science, vol. 321, pp. 385-388, 2008.

[3] D. B. Zhang, E. Akatyeva, and T. Dumitrică, "Bending Ultrathin Graphene at the Margins of Continuum Mechanics," Physical Review Letters, vol. 106, p. 255503, 2011.

[4] R. Xu, Y. Wang, B. Liu, and D. Fang, "Mechanics Interpretation on the Bending Stiffness and Wrinkled Pattern of Graphene," Journal of Applied Mechanics, vol. 80, p. 040910, 2013.
[5] M. Poot and H. S. J. van der Zant, "Nanomechanical properties of few-layer graphene membranes," Applied Physics Letters, vol. 92, pp. -, 2008 .

[6] W. H. Duan and C. M. Wang, "Nonlinear bending and stretching of a circular graphene sheet under a central point load," Nanotechnology, vol. 20, p. 075702, 2009.

[7] R. Rasuli, A. I. zad, and M. M. Ahadian, "Mechanical properties of graphene cantilever from atomic force microscopy and density functional theory," Nanotechnology, vol. 21, p. 185503, 2010.

[8] Y. Wang, S. W. Tong, X. F. Xu, B. Özyilmaz, and K. P. Loh, "Interface Engineering of Layer by Layer Stacked Graphene Anodes for High Performance Organic Solar Cells," Advanced Materials, vol. 23, pp. 1514-1518, 2011. 